HEP-TH/0510171

NORDITA-2005-67

\title{
Wrapping interactions and a new source of corrections to the spin-chain/string duality
}

\author{
J. Ambjørn ${ }^{a, d}$, R. A. Janik ${ }^{b}$ and C. Kristjansen ${ }^{c}$ \\ ${ }^{a}$ The Niels Bohr Institute, Copenhagen University \\ Blegdamsvej 17, DK-2100 Copenhagen Ø, Denmark. \\ email: ambjorn@nbi.dk \\ ${ }^{b}$ Institute of Physics, Jagellonian University, \\ Reymonta 4, PL 30-059 Krakow, Poland. \\ email: romuald@th.if.uj.edu.pl \\ ${ }^{c}$ NORDITA, \\ Blegdamsvej 17, DK-2100 Copenhagen Ø, Denmark. \\ email kristjan@nbi.dk \\ ${ }^{d}$ Institute for Theoretical Physics, Utrecht University, \\ Leuvenlaan 4, NL-3584 CE Utrecht, The Netherlands.
}

\begin{abstract}
Assuming that the world-sheet sigma-model in the AdS/CFT correspondence is an integrable quantum field theory, we deduce that there might be new corrections to the spin-chain/string Bethe ansatz paradigm. These come from virtual particles propagating around the circumference of the cylinder and render Bethe ansatz quantization conditions only approximate. We determine the nature of these corrections both at weak and at strong coupling in the near BMN limit, and find that the first corrections behave qualitatively as wrapping interactions at weak coupling.
\end{abstract}




\section{Introduction}

The discovery of integrable structures underlying planar $\mathcal{N}=4$ SYM $[1,2$, $3,4]$ as well as classical string theory on $A d S_{5} \times S^{5}[5,6,7,8,9,10,11]$ has opened up new avenues for testing the AdS/CFT correspondence [12]. Moreover, the application of spin chain techniques has facilitated the analysis on the gauge theory side. Non-trivial comparisons of gauge theory anomalous dimensions and string state energies can now be carried out in three different regimes: The BMN limit [13], the near BMN limit [14] and the spinning string limit [15]. Whereas for the first of these three limits everything at the level of anomalous dimensions appears to work fine the latter two are plagued by vexing three-loop discrepancies [16, 17].

It has been suggested that the three-loop discrepancy for near BMN states and spinning strings is due to the non-commutativity of the two limits which are involved in performing the analysis and which are imposed in different order on respectively the string and gauge theory side [17]. To perform an analysis on the gauge theory side in which the order of limits would be the same as on the string theory side one would have to take into account so-called wrapping interactions [18]. In the spin chain language wrapping interactions are interactions whose range exceeds the length of the chain. It is a fact that wrapping interactions are important when it comes to the determination of anomalous dimensions of short operators. For instance, wrapping interactions contribute to the four-loop anomalous dimension of the Konishi operator $[2,19]$. A systematic discription of wrapping interactions in terms of Feynman diagrams can be found in [20]. An alternative explanation of the three-loop discrepancy was suggested in [21].

On the gauge theory side it is very hard to do any rigorous derivations beyond the two and three loop ones of [2, 22], see also [23]. However, assuming integrability and BMN scaling at higher loop orders a conjecture for an all loop Bethe ansatz has been put forward [18, 24, 25]. On the string theory side it is possible to derive integral equations encoding all classical string solutions $[8,26,10,11]$. These integral equations can be viewed as classical, continuum Bethe equations. Inspired by these two achievements a suggestion for a Bethe ansatz for quantum strings was presented in [27, 24, 25]. The quantum string Bethe ansatz is identical to the gauge theory one up to two loop order but differs from it beyond two loops. The all loop gauge theory Bethe ansatz and the quantum string one are very similar in nature. They both express the condition for factorized scattering for a set of elementary 
excitations with individual momenta $p_{k}$

$$
\exp \left(i L p_{k}\right)=\prod_{j \neq k}^{M} S\left(p_{k}, p_{j}\right)
$$

where $S$ is the $S$-matrix for scattering of two of these excitations and $L$ is the length of the spin chain. The elementary excitations have the same dispersion relation on the gauge and string theory side. What differs between the two sides is the form of the $S$-matrices. The difference can conveniently be encoded in a so-called dressing factor

$$
S^{\text {string }}=\hat{S}^{\text {dressing }} S^{\text {gauge }},
$$

which can be expressed as a phase shift, i.e.

$$
\hat{S}^{\text {dressing }}\left(p_{k}, p_{j}\right)=\exp \left(i \theta\left(p_{k}, p_{j}\right)\right)
$$

where

$$
\theta\left(p_{k}, p_{j}\right)=2 \sum_{r=2}^{\infty} c_{r}(\lambda)\left(\frac{\lambda}{16 \pi^{2}}\right)^{r}\left(q_{r}\left(p_{k}\right) q_{r+1}\left(p_{j}\right)-q_{r+1}\left(p_{k}\right) q_{r}\left(p_{j}\right)\right),
$$

with $\lambda$ being the 't Hooft coupling constant and the $q_{r}$ 's certain conserved charges $[18,27]$. The expansion coefficients $c_{r}(\lambda)$ must fulfill that $c_{r}(\lambda) \rightarrow 1$ as $\lambda \rightarrow \infty$ in order that the classical string theory limit is correctly reproduced. Recently, it was found by a study of one-loop string sigma model corrections in the $\operatorname{sl}(2)$ sector that it is not possible to have $c_{r}(\lambda)=1$ for all values of $\lambda[28]$. The first string sigma model loop correction produces contributions to energies of strings spinning with total angular momentum $L$ which contain half integer powers of $\lambda^{\prime}=\frac{\lambda}{L^{2}}[29,30]$ starting at order $\left(\lambda^{\prime}\right)^{5 / 2}[30]$ as well as non-perturbative contributions containing factors of the type $\exp \left(-\frac{1}{\sqrt{\lambda^{\prime}}}\right)[29]$. The appearance of terms of the type $\exp \left(-\frac{1}{\sqrt{\lambda^{\prime}}}\right)$ as well as terms with half-integer powers of $\lambda^{\prime}$ was earlier observed in the BMN limit in [31] in connection with a study of the three string interaction vertex. The leading half integer power of $\lambda^{\prime}$ in string energies can be accounted for by the phase factor $(4)$ if the coefficient $c_{2}(\lambda)$ has the expansion [30]

$$
c_{2}(\lambda)=1-\frac{3}{4} \frac{1}{\sqrt{\lambda}}+\mathcal{O}\left(\frac{1}{\lambda}\right) .
$$


This opens the interesting possibility that $c_{2}(\lambda)$ could tend to zero at weak coupling [30] and thus that the three loop discrepancy for near BMN states as well as for spinning strings could indeed be due to an order of limits problem. Recently, it was shown that it is possible to obtain the classical string equations of motion of the $\mathrm{su}(2)$ sector as the classical limit of an integrable quantum field theory, namely the $O s p(2 m+2 \mid 2 m)$ coset model [32]. This model is defined on the plane and not on the cylinder as needed for a quantum string theory on $A d S_{5} \times S^{5}$. The current understanding is that the $O s p(2 m+2 \mid 2 m)$ coset model is capable of capturing the quantum effects introducing half-integer powers of $\lambda$ in the expansions of string energies but not the finite size corrections which appear when the theory is put on a cylinder [32].

In the present paper we will explore the consequences of putting an integrable quantum field theory (IQFT) on a cylinder. In particular we shall discuss what happens if the elementary excitations of the field theory in stead of the standard relativistic dispersion relation obey the dispersion relation implied by the conjectured quantum string Bethe equations [27]. We shall work in the near BMN limit and shall show that wrapping interactions generically give rise to contributions of order $\lambda^{L}$ at weak coupling and of order $\exp \left(-\frac{1}{\sqrt{\lambda^{\prime}}}\right)$ at strong coupling.

One of the established properties of an integrable quantum field theory on a cylinder is the fact that the Bethe ansatz quantization conditions

$$
e^{i p_{k} L}=\prod_{j \neq k} S\left(p_{k}, p_{j}\right)
$$

are no longer exact (see e.g. [33] section 5). The effect is very generic and comes from virtual corrections - excitations going around the circumference of the cylinder (see fig. 1 below).

Intuitively such processes, involving a virtual particle going around the cylinder, when translated into Feynman graphs of the gauge theory, should correspond to wrapping interactions. These types of virtual effects can be described $^{1}$ through S-matrix information, where the S-matrix is defined in the infinite volume limit. Thus, since there are proposals for the asymptotic S-matrix for gauge theory/strings we propose to interpret them as the infinite volume scattering data and use the framework of the virtual corrections to IQFT to incorporate the effect of wrapping interactions. We show that at

\footnotetext{
${ }^{1}$ At leading order in all relativistic IQFT's, and for some specific theories exactly.
} 
weak coupling the order at which the virtual corrections set in is $\lambda^{L}$ just as expected for wrapping interactions.

The outline of this paper is as follows. In section 2 we will review these corrections in ordinary relativistic integrable field theories. In section 3 we will motivate what changes need to be done in order to incorporate the nonstandard dispersion relation characteristic of excitations in the conjectured long range Bethe ansatz, then in section 4 we estimate the size of the corrections both at weak coupling and in the near-BMN limit. We close the paper with a summary and outlook.

\section{Finite size mass shift in relativistic inte- grable field theories}

In $[34,35]$ Lüscher calculated the leading order corrections to the (infinite volume) masses/energies of 1-particle states when put on a cylinder of circumference $L$ :

$$
m(L)=m(L=\infty)+\Delta m_{\mu}(L)+\Delta m_{F}(L) .
$$

These corrections arise from two different types of processes: the first is the so-called $\mu$-term which arises when the particle splits into a pair of virtual on-shell particles which go around the cylinder and recombine later, while the second one, the $F$-term comes from a virtual particle loop where the virtual particle goes around the circumference of the cylinder (see fig. 1).

The expressions for these terms in the case of $1+1$ dimensions and a single mass scale in the theory are [38]

$$
\begin{aligned}
\frac{\Delta m_{\mu}(L)}{m(\infty)} & =-\frac{\sqrt{3}}{2} \sum_{b, c} M_{a b c}(-i) \operatorname{res}_{\theta=2 \pi i / 3} S_{a b}^{a b}(\theta) \cdot e^{-\frac{\sqrt{3}}{2} m L} \\
\frac{\Delta m_{F}(L)}{m(\infty)} & =-\int_{-\infty}^{\infty} \frac{d \theta}{2 \pi} e^{-m L \cosh \theta} \cosh \theta \sum_{b}\left(S_{a b}^{a b}\left(\theta+i \frac{\pi}{2}\right)-1\right)
\end{aligned}
$$

where $S_{a b}^{a b}(\theta)$ is the (infinite volume) $S$-matrix, and $M_{a b c}=1$ if $c$ is a bound state of $a$ and $b$ and zero otherwise. These formulas have been checked to agree with a wide variety of examples such as Ising field theory, the so-called scaling Lee-Yang model (SLYM), various perturbed minimal model CFT's etc. $[37,38]$. 

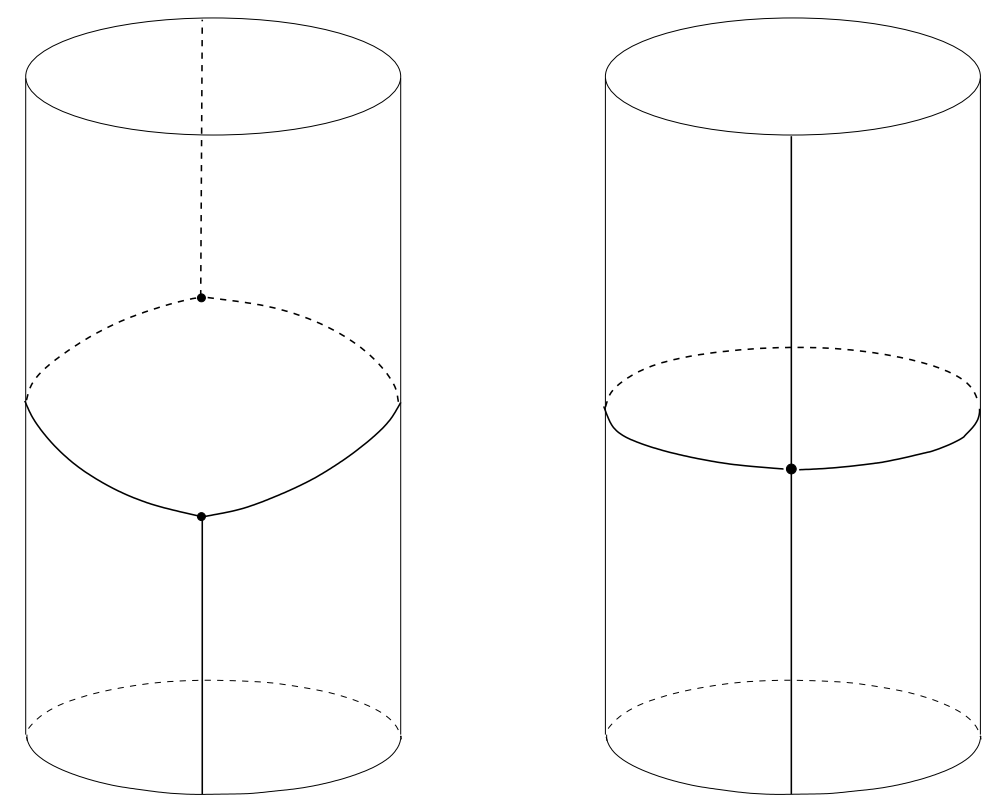

Figure 1: The diagram to the left (the $\mu$-term) shows a particle splitting in two virtual, on-shell particles, traveling around the cylinder and recombining. The diagram to the right (the F-term) shows a virtual particle going around the circumference of the cylinder.

However, it is rather difficult to use these formulas directly in the case of the world sheet integrable field theories appearing in our present context. The proofs of Lüscher's formulas were a mixture of diagrammatic analysis and analytical continuation where Lorentz invariance was crucial (although clearly many aspects remain valid on the lattice [39]). In particular, one always had the dispersion relation $E^{2}-p^{2}=m^{2}$ and the parametrization of energy and momentum through rapidity

$$
E=m \cosh \theta, \quad p=m \sinh \theta .
$$

For the world sheet string theories in curved AdS space-time it was pointed out $[40,14,16,9]$ that the usual light cone gauge like $\tau=t$ (where $t$ is e.g. the global AdS time) is inconsistent with putting a Minkowskian metric on the world sheet. Hence the second gauge condition is usually taken to be the uniform gauge where the density of a conserved charge (such as the R-charge $J)$ is spread uniformly along the string ${ }^{2}$. Then the gauge-fixed field theory

\footnotetext{
${ }^{2}$ Usually one makes the condition $p_{\phi}=J$ keeping the size of the cylinder to $2 \pi$ [9]. But
} 
is not explicitly Lorentz-invariant! A common ingredient of the asymptotic Bethe ansätze is the dispersion relation for all elementary excitations (here $E \equiv \Delta-J)$ :

$$
E=\sqrt{1+8 g^{2} \sin ^{2} \frac{p}{2}}, \quad g^{2}=\frac{\lambda}{8 \pi^{2}} .
$$

In the following we will assume that this is indeed the true dispersion relation for excitations in the integrable quantum field theories on the world sheet and explore the consequences of this assumption.

Another point which one should mention is that e.g. in the near BMN limit the zero momentum 1-particle states are protected by supersymmetry and what one is really interested in are corrections to 2-particle (or higher) states for which we lack similar expressions.

In order to attack the above problems it is convenient to look at a way in which the finite size virtual corrections can be calculated exactly, and which may shed more light on the origin of the formulas (8) and (9).

There are basically three (interrelated) ways of computing exactly the spectrum of an integrable QFT on a cylinder: the Thermodynamic Bethe Ansatz (TBA) [42], Nonlinear Integral equation (NLIE) or Destri-de-Vega (DDV) equation [43], and functional relations [33]. All this is still an area of active research [44]. We will here concentrate on the first of these approaches.

Before we start let us emphasize that the Thermodynamic Bethe Ansatz that we consider here is not the thermodynamic approximation which is used for approximately solving Algebraic Bethe Ansatz equations for a large number of roots in the spin chain/string literature. We use the term in the sense that it is used in the context of relativistic integrable field theories.

\section{Thermodynamic Bethe Ansatz}

The Thermodynamical Bethe Ansatz [42] was originally devised as a method for finding how the ground state of an integrable field theory depends on the circumference $L$. The main idea is to consider the theory on a very elongated torus with circumference $L$ and length $R \rightarrow \infty$, and to compute the (euclidean) partition function. From the point of view of $L$ being space and

this populates the action with explicit factors of $J$. It is more convenient for our purposes to set the density to be equal to 1 and then the total R-charge is encoded just in the size of the cylinder like in [41]. 
$R$ being very large 'time' this gives the ground state energy being calculated:

$$
E_{0}(L)=-\frac{1}{R} \log Z .
$$

On the other hand if one looks at $R$ being space and $L$ being the (euclidean) time this is just the thermal free energy of the system on an infinite line, which can be calculated by minimising $E-T S$ with $T=1 / L$. The main point is that for the theory with $R \rightarrow \infty$, Bethe ansatz quantization remains exact. Then one introduces continuous densities for the occupied roots AND appropriate entropy factors. The result is the set of equations ${ }^{3}$ :

$$
\begin{aligned}
\varepsilon(\theta) & =L E_{T B A}(\theta)-\phi * L, \\
E_{0} & =\int_{-\infty}^{\infty} \frac{d \theta}{2 \pi} p_{T B A}^{\prime}(\theta) L(\theta),
\end{aligned}
$$

where

$$
\begin{aligned}
L(\theta) & =\log \left(1+e^{-\varepsilon(\theta)}\right), \\
(\phi * L)(\theta) & =\int_{-\infty}^{\infty} \frac{d \theta^{\prime}}{2 \pi} \phi\left(\theta-\theta^{\prime}\right) L\left(\theta^{\prime}\right), \\
\phi(\theta) & =-i \frac{d}{d \theta} \log S(\theta),
\end{aligned}
$$

and $E_{T B A}(\theta)=m \cosh \theta$, and $p_{T B A}(\theta)=m \sinh \theta$.

As it stands the Thermodynamic Bethe Ansatz seems to be confined to being a tool for finding just the ground state energy. However, in [45] Dorey and Tateo suggested that by analytical continuation one could obtain the energies of excited states. This program was carried out first in the scaling Lee-Yang model (SLYM) [45] and then in a series of perturbed minimal model CFT's [46].

The underlying mechanism of the construction is that $L(\theta)=\log (1+$ $\left.e^{-\varepsilon(\theta)}\right)$ developed singularities which after a contour deformation added source terms to the right hand sides of equations (13) and (14). E.g. for a 1-particle state at zero momentum, the $m L \cosh \theta$ in (13) should be substituted by

$$
m L \cosh \theta+\log \frac{S\left(\theta-\theta_{0}\right)}{S\left(\theta+\theta_{0}\right)},
$$

\footnotetext{
${ }^{3}$ For the simplest case of a single particle in the spectrum.
} 
where $\theta_{0}$ is a singularity of $L(\theta)$, which leads to an additional equation $\varepsilon\left(\theta_{0}\right)=i \pi$. This set of equations then gives exact results for the excited state energy $E_{1}(L)$ for any $L$. Moreover, Lüscher's formulas for (8) and (9) then immediately follow from solving that system of equations by iteration (see $[45])$.

For our purposes it is interesting to obtain the form of corrections for a 2-particle state for which we lacked an explicit expression.

From the results in [45] we obtain

$$
\begin{aligned}
E= & 2 \cosh \theta_{B}+2 \cosh \theta_{B} \cdot \delta- \\
& \int_{-\infty}^{\infty} \frac{d \theta}{2 \pi} \cosh \theta e^{-L \cosh \theta} S\left(\theta+i \frac{\pi}{2}-\theta_{B}\right) S\left(\theta+i \frac{\pi}{2}+\theta_{B}\right),
\end{aligned}
$$

where $\theta_{B}$ is the Bethe root which follows from solving the Bethe quantization condition

$$
e^{i L \sinh \theta_{B}}=S\left(2 \theta_{B}\right) .
$$

We see that the first piece of (19) is the classical energy of the 2-particle state. The second piece is a $\mu$-term of the form

$$
\delta=3 e^{-\frac{\sqrt{3}}{2} L \cosh \theta_{B}} \operatorname{coth} \theta_{B} \sqrt{\frac{2 \cosh 2 \theta_{B}+1}{2 \cosh 2 \theta_{B}-1}},
$$

while the last term is the analogue of the $F$-term. The exponential factor remains the same, while the two S-matrices correspond to a virtual particle of rapidity $\theta+i \pi / 2$ moving around the cylinder and scattering off the two particles with Bethe roots (rapidities) $\pm \theta_{B}$.

The point that we want to make here is that the structure of the $F$-term correction to the 2-particle state is a straightforward generalization of the Lüscher mass shift formula for a 1-particle state. In view of applications to the world sheet theory we would expect a first nontrivial correction to occur at the level of 2-particle states since the mass of the one-particle states are protected by supersymmetry. The absence of corrections for the ground state and 1-particle states should be a property of the full world sheet theory and cannot be obtained just in e.g. the $s u(2)$ sector. The reason is that the $\mathrm{su}(2)$ excitation scatters non-trivially from other excitations [25] which might therefore circulate in the loop.

Another motivation for discussing at length the TBA derivation of Lüscher's formulas is that the space-time interpretation of the derivation of TBA may 
suggest its generalization to the case of the world sheet theory which, when gauge-fixed, is no longer Lorentz-invariant.

In the next section we will use the TBA intuition to propose what changes are needed when the dispersion relation is no longer exactly relativistic but is modified to (11). We will also try to motivate, using a solvable example, that such a procedure can be legitimate - which is of course not completely clear a-priori.

\section{Nonstandard dispersion relations}

As we saw above, the derivation of the TBA is based on a modular transformation on the torus i.e. an interchange of space and time. For a relativistic theory this does not really make a difference but it is far from clear if such a philosophy may be applied at all to the rather nonstandard world-sheet theories.

In order to motivate this we will show an example where the proposed procedure can be proved to give the exact result despite the fact that the dispersion relation is not exactly relativistic.

\section{The Ising model off criticality}

This example has been given by Lüscher in his Cargese lectures [34]. We need to be here more explicit in the calculation, as the intermediate steps will be crucial for our purposes. The Ising model on a finite $L \times L$ lattice can be completely solved in terms of transfer matrices which can be expressed through free fermion operators with the dispersion relation [36]:

$$
\cosh E_{q}=\cosh m_{0}+1-\cos q \equiv \cosh m_{0}+2 \sin ^{2} \frac{q}{2} .
$$

The mass gap (i.e. the mass/energy of the lowest lying excitation) can be expressed through an exact formula:

$$
m(L)=m_{0}+\frac{1}{2} \sum_{\nu=0}^{L-1} E_{\frac{\pi}{L}(2 \nu+1)}-\frac{1}{2} \sum_{\nu=0}^{L-1} E_{\frac{\pi}{L} \cdot 2 \nu}=m_{0}-\frac{1}{2} \sum_{\nu=0}^{2 L-1} e^{i \pi \nu} E_{\frac{\pi}{L} \cdot \nu} .
$$

The discrete sum can be rewritten using the (finite) Poisson resummation formula [47] as

$$
m(L)=m_{0}-L \sum_{\nu=-\infty}^{\infty} \int_{-\pi}^{\pi} \frac{d q}{2 \pi} e^{i q L(2 \nu+1)} E_{q}
$$


Now after an integration by parts one changes variables to

$$
Q=-i E_{q},
$$

$Q$ will play the role of the new momentum in what follows. For integer $L$ one then gets

$$
m(L)=m_{0}+\sum_{\nu=-\infty}^{\infty} \frac{1}{2 \nu+1} \int_{-i E_{-\pi}}^{-i E_{\pi}} \frac{d Q}{2 \pi} e^{i q(Q) L(2 \nu+1)} .
$$

We note that due to the form of $(22) q(Q)=i E_{Q}$ so the exponent simplifies to $-E_{Q} L(2 \nu+1)$. After a slightly nontrivial contour argument it turns out that one can change the contour of integration to go from $-\pi$ to $\pi$ just as if $Q$ was a physical momentum. One then gets

$$
m(L)=m_{0}+\sum_{\nu=0}^{\infty} \frac{2}{2 \nu+1} \int_{-\pi}^{\pi} \frac{d Q}{2 \pi} e^{-L(2 \nu+1) E_{Q}} \sim m_{0}+2 \int_{-\pi}^{\pi} \frac{d Q}{2 \pi} e^{-L E_{Q}}
$$

The leading correction is then the term with $\nu=0$. The exponent is just as in the formula for relativistic $F$-term correction (with the $\mathrm{S}$ matrix equal to -1 , which is the value for the massive deformation of the Ising model).

\section{Space-time interchange}

Let us now go back and analyze what kind of analytical continuations were made here and adopt a notation more reminiscent of the TBA expressions. What plays the role of momentum in the final formula (27) is

$$
p_{T B A} \equiv Q=-i E_{q},
$$

while the energy appearing in the exponent of $(27)$ is in fact $-i$ times the original momentum expressed in terms of $Q \equiv p_{T B A}$ :

$$
E_{T B A}=-i q(Q) \text {. }
$$

The above substitutions are indeed quite natural from the point of view of a modular transformation exchanging space and time. Since time should be interchanged with space one should Wick rotate both coordinates (hence the $-i$ 's in (28) and (29)), and also exchange $E$ and $q$ with each other. In the final integral (27) $p_{T B A} \equiv Q$ is taken to be real. 
To add some more plausibility to the interpretation of the above formulas, one sees that in the Lorentz invariant case the dispersion relation in the double Wick rotated space remains the same and the shift of the rapidity of the virtual particle by $i \pi / 2$ in the $F$-term exactly corresponds to the above transformations (28)-(29).

$$
m \cosh (\theta+i \pi / 2)=i m \sinh \theta, \quad m \sinh (\theta+i \pi / 2)=i m \cosh \theta .
$$

In the following section we will apply these ideas to estimate the size of the correction terms for the world sheet theory with the dispersion relation (11) but before we do that, we will show that the above procedure works for a modified Ising model with (11).

\section{Modified Ising model}

Since we lack any explicit example of a completely defined integrable theory with the dispersion relation (11), we will construct a somewhat artificial but exactly solvable example. Let us modify the Ising model defined by the transfer matrices involving free fermions by changing their dispersion relation from (22) to (11).

The exact mass shift can then be obtained in exactly the same fashion. It is given by formulae analogous to (23)-(24), i.e.

$$
m(L)=1-L \sum_{\nu=-\infty}^{\infty} \int_{-\pi}^{\pi} \frac{d q}{2 \pi} e^{i q L(2 \nu+1)} \sqrt{1+8 g^{2} \sin ^{2} \frac{q}{2}} .
$$

Let us now evaluate $E_{T B A}\left(p_{T B A}\right)$. Performing the substitutions (28)-(29) we obtain

$$
E_{T B A}\left(p_{T B A}\right)=2 \operatorname{arcsinh}\left(\frac{1}{2 \sqrt{2} g} \cdot \sqrt{1+p_{T B A}^{2}}\right) .
$$

Now again the quite remarkable identity holds for integer $L$ :

$$
\begin{aligned}
&-L \int_{-\pi}^{\pi} \frac{d q}{2 \pi} e^{i q L(2 \nu+1)} \sqrt{1+8 g^{2} \sin ^{2} \frac{q}{2}} \\
&=\frac{1}{2 \nu+1} \int_{-\infty}^{\infty} \frac{d q}{2 \pi} e^{-L(2 \nu+1) \cdot 2 \operatorname{arcsinh}\left(\frac{\sqrt{1+p_{T B A}^{2}}}{2 \sqrt{2} g}\right),}
\end{aligned}
$$

which for $\nu=0$ reproduces the expected correction

$$
m(L)=1+2 \int_{-\infty}^{\infty} \frac{d q}{2 \pi} e^{-L \cdot 2 \operatorname{arcsinh}\left(\frac{\sqrt{1+p_{T B A}^{2}}}{2 \sqrt{2} g}\right)}
$$


but with the somewhat odd-looking expression (32) for the energy.

\section{$4 \quad$ Wrapping interactions and near-BMN limit}

Let us now apply the above considerations to the case of the world sheet theory with the dispersion relation (11). We will evaluate at what order does the virtual correction enter both at weak and at strong gauge theory coupling (in the near BMN limit). We will not try here to obtain the exact form of the correction since this would have to involve the full world sheet theory whose S-matrix is so far not known completely. Moreover the scattering is non-diagonal making the procedure even more complicated. The conjectured Bethe ansätze [25] may be used in this respect but carrying out this program still remains a very nontrivial task which we leave for future investigation.

In the following we will discuss first the $F$-term and then the $\mu$-term.

\section{The F-term}

The size of the $F$ term at large $L$ is governed by the exponential factor

$$
e^{-L E_{T B A}}=e^{-L \cdot 2 \operatorname{arcsinh}\left(\frac{\sqrt{1+p_{T B A}^{2}}}{2 \sqrt{2} g}\right)} .
$$

Let us see how this expression behaves at small coupling. Then the argument of the arcsinh is very large and we may substitute it by a logarithm $E_{T B A} \sim$ $-2 \log g+\ldots$ Consequently the correction term is of the order

$$
g^{2 L}
$$

which is exactly the expected order when wrapping interactions should set in. This further supports the intuition that virtual corrections in the world sheet theory corresponding to particles moving in loops around the cylinder should correspond to wrapping interactions on the gauge theory side.

Let us now proceed to take the near BMN limit. We take ${ }^{4} L \sim J \rightarrow \infty$ but at the same time keep $\lambda^{\prime}=\lambda / J^{2}$. Therefore

$$
8 g^{2}=\frac{\lambda^{\prime} J^{2}}{\pi^{2}}
$$

\footnotetext{
${ }^{4}$ For a two impurity state in the $\mathrm{su}(2)$ sector $L=J+2$.
} 
In this limit the argument of the arcsinh is small and the exponent (35) governing the size of the corrections takes the form ${ }^{5}$

$$
e^{-\frac{2 \pi}{\sqrt{\lambda^{\prime}}} \sqrt{1+p_{T B A}^{2}}}
$$

\section{$\mu$-term}

We have much less control over the precise form of the $\mu$-term, however its physical origin is quite clear. It occurs when there is a possible process when a particle can decay into a pair of virtual particles which are on-shell (but yet will have in general complex momenta).

For Lorentz-invariant theories, the exponents of the $\mu$-terms (8) and (21) are seen to follow from the imaginary parts of the momenta of the two virtual particles. Explicitly, suppose that an on-shell particle of momentum $p_{0}$ disintegrates into a pair of particles with momenta $p_{1}$ and $p_{2}$ which are on-shell. From the kinematics we obtain for $p_{1,2}$ :

$$
p_{1,2}=\frac{p_{0}}{2} \pm i \frac{\sqrt{3}}{2} \sqrt{1+p_{0}^{2}}
$$

We do not have a precise expression for the $\mu$-term in the case of multiparticle states. We will here just use an ad-hoc formula $\exp \left(-L \cdot \operatorname{Im} p_{i}\right)$, and we find that it reproduces the exponents in (8) and (21).

Repeating the same kind of calculation with the dispersion relation (11) gives in general quite complicated expressions. We may however take $p_{0}$ to be the momentum of a particle in a 2-particle state in the near BMN limit:

$$
p_{0}=\frac{2 \pi n}{J+2}+\frac{2 \pi n}{\sqrt{1+\lambda^{\prime} n^{2}}(J+2)^{2}}+\ldots
$$

Assuming $p_{i}$ to be small we obtain

$$
p_{1,2}=\frac{1}{J}\left(n \pi \pm i \frac{\sqrt{3}}{\sqrt{\lambda^{\prime}}} \pi \sqrt{1+\lambda^{\prime} n^{2}}\right) .
$$

This suggests that the $\mu$-term, if present, would also give $1 / \sqrt{\lambda^{\prime}}$ corrections in the near BMN limit.

\footnotetext{
${ }^{5}$ We note that in this limit the same result would also arise from an unmodified BMN dispersion relation $E=\sqrt{1+2 g^{2} p^{2}}$.
} 


\section{Conclusions}

Lacking a direct proof of the AdS/CFT correspondence, tests which are truly reflecting non-trivial interactions are of utmost importance. The integrable structures discovered both at the string side and the $\mathcal{N}=4$ SYM side provide a good testing ground and there has been extensive research comparing the predictions coming from the string world-sheet sigma model with the predictions from the spin-chain model of $\mathcal{N}=4 \mathrm{SYM}$.

Numerous examples of very detailed agreement between gauge and string theory have been found, nevertheless there remains some subtle but significant discrepancies. One notable example is the notorious three loop discrepancy occurring both in the near-BMN limit and for spinning strings. Another is the appearance half-integer powers of $\lambda$ in string sigma model loop corrections, cf. the introduction. However, it is known that at least some ingredients have not been included in the asymptotic Bethe ansätze such as e.g. gauge theory wrapping interactions.

While integrability on the string theory side is fairly developed for the classical sigma model not much is known for the complete quantum theory. A very recent attempt to study the quantum effects via S-matrix (Bethe Ansatz) techniques can be found in the paper by Mann and Polchinski [32], who considered directly a relativistic quantum field theory on $R^{2}$ (a $O S p(2 m+2 \mid 2 m)$ supercoset model) with a known S-matrix. They found an embedding of the 'classical' string $\mathrm{su}(2)$ sector into the Bethe ansatz of their full quantum theory, and found generic corrections of the type $1 / \sqrt{\lambda}$, but did not consider finite size effects.

In our paper we wanted to concentrate on the specific features of an integrable quantum field theory when put on a cylinder, as is always ultimately required for the closed string worldsheet theory. The generic feature is then that the Bethe ansatz quantization condition is no longer exact and receives virtual corrections from particles moving in loops around the circumference of the cylinder.

The energy shifts due to these effects in a finite volume are most conveniently calculated using the Thermodynamic Bethe Ansatz. ${ }^{6}$ We provided arguments that despite the non-Lorentz-invariant nature of the dispersion relation (11) conjectured to hold for the excitations of the $A d S$ string, one

\footnotetext{
${ }^{6}$ Although no general proof of this method for excited states exists, extensive tests have been performed in various relativistic integrable field theories [45, 46, 48].
} 
can still use TBA. It does not seem possible at this stage to perform a complete calculation for the world-sheet theory. Therefore, in this paper we have confined ourselves to understand just the general nature of the corrections, using only the dispersion relation (11) as an assumption.

At weak coupling we find that the first corrections are of the order $\lambda^{L}$. It is natural to conjecture that the analogous term on the gauge-theory side is due to wrapping interactions in the spin-chain picture. This is also very intuitive as the propagation of a virtual excitation around the cylinder would presumably, when translated somehow into gauge theory Feynman graphs, be described by graphs 'wrapping' the cylinder.

At strong coupling the contribution is of the order $e^{-1 / \sqrt{\lambda^{\prime}}}$ which cannot be seen directly in perturbative gauge theory. However, as mentioned earlier, in string sigma model loop corrections one has found contributions to string energies involving half integer powers of $\lambda^{\prime}$ as well as contributions of of the form $e^{-1 / \sqrt{\lambda^{\prime}}}[28,29,30]$, see also [31].

It would be very interesting to perform a more complete calculation, perhaps using all or a part of the asymptotic Bethe ansätze of [25]. The same could be also considered in the supercoset model of [32], which has the additional simplification that it is a true relativistic integrable field theory so it does not suffer from the conceptual difficulties associated with the nonstandard dispersion relation. Last but not least, it would be very interesting to try to extend the formalism of such finite size virtual corrections to many particle states dual to macroscopic spinning strings.

Acknowledgments. The authors were all supported by ENRAGE (European Network on Random Geometry), a Marie Curie Research Training Network financed by the European Community's Sixth Framework Programme, network contract MRTN-CT-2004-005616. RJ thanks the NBI for hospitality when this work was carried out. RJ was supported in part by Polish Ministry of Science and Information Society Technologies grants 1P03B02427 (2004-2007) and 1P03B04029 (2005-2008).

\section{References}

[1] J. A. Minahan and K. Zarembo, "The Bethe-ansatz for $\mathrm{N}=4$ super Yang-Mills," JHEP 0303, 013 (2003), hep-th/0212208. 
[2] N. Beisert, C. Kristjansen and M. Staudacher, "The dilatation operator of N = 4 super Yang-Mills theory," Nucl. Phys. B 664 (2003) 131, hepth/0303060.

[3] N. Beisert and M. Staudacher, "The N = 4 SYM integrable super spin chain," Nucl. Phys. B 670 (2003) 439, hep-th/0307042.

[4] A. V. Belitsky, S. E. Derkachov, G. P. Korchemsky and A. N. Manashov, "Quantum integrability in (super) Yang-Mills theory on the light-cone," Phys. Lett. B 594 (2004) 385 [arXiv:hep-th/0403085].

[5] I. Bena, J. Polchinski and R. Roiban, "Hidden symmetries of the AdS(5) x S**5 superstring," Phys. Rev. D 69 (2004) 046002, hep-th/0305116.

[6] G. Arutyunov, S. Frolov, J. Russo and A. A. Tseytlin, "Spinning strings in $\operatorname{AdS}(5) \times \mathrm{S}^{* *} 5$ and integrable systems," Nucl. Phys. B 671 (2003) 3, hep-th/0307191; G. Arutyunov, J. Russo and A. A. Tseytlin, "Spinning strings in $\operatorname{AdS}(5) \times \mathrm{S}^{* *}$ 5: New integrable system relations," Phys. Rev. D 69 (2004) 086009, hep-th/0311004.

[7] L. Dolan, C. R. Nappi and E. Witten, "A relation between approaches to integrability in superconformal Yang-Mills theory," JHEP 0310 (2003) 017, hep-th/0308089.

[8] V. A. Kazakov, A. Marshakov, J. A. Minahan and K. Zarembo, "Classical / quantum integrability in AdS/CFT," JHEP 0405 (2004) 024, hep-th/0402207.

[9] G. Arutyunov and S. Frolov, "Integrable Hamiltonian for classical strings on AdS(5) x S**5," JHEP 0502, 059 (2005), hep-th/0411089.

[10] N. Beisert, V. A. Kazakov, K. Sakai and K. Zarembo, "The algebraic curve of classical superstrings on $\operatorname{AdS}(5) \times \mathrm{S}^{* *} 5$," hep-th/0502226.

[11] L. F. Alday, G. Arutyunov and A. A. Tseytlin, "On integrability of classical superstrings in $\operatorname{AdS}(5) \times \mathrm{S}^{* *}$," JHEP 0507 (2005) 002, hepth/0502240.

[12] J. M. Maldacena, "The large N limit of superconformal field theories and supergravity," Adv. Theor. Math. Phys. 2 (1998) 231 [Int. J. Theor. Phys. 38 (1999) 1113], hep-th/9711200; S. S. Gubser, I. R. Klebanov and 
A. M. Polyakov, "Gauge theory correlators from non-critical string theory," Phys. Lett. B 428 (1998) 105, hep-th/9802109; E. Witten, "Antide Sitter space and holography," Adv. Theor. Math. Phys. 2 (1998) 253, hep-th/9802150.

[13] D. Berenstein, J. M. Maldacena and H. Nastase, "Strings in flat space and pp waves from $\mathrm{N}=4$ super Yang Mills," JHEP 0204 (2002) 013, hep-th/0202021.

[14] C. G. . Callan, H. K. Lee, T. McLoughlin, J. H. Schwarz, I. J. Swanson and $\mathrm{X}$. Wu, "Quantizing string theory in $\operatorname{AdS}(5) \times \mathrm{S}^{* *} 5$ : Beyond the pp-wave," Nucl. Phys. B 673 (2003) 3, hep-th/0307032.

[15] S. Frolov and A. A. Tseytlin, "Semiclassical quantization of rotating superstring in AdS(5) x S(5)," JHEP 0206 (2002) 007, hep-th/0204226; S. Frolov and A. A. Tseytlin, "Quantizing three-spin string solution in AdS(5) x S**5," JHEP 0307 (2003) 016, hep-th/0306130.

[16] C. G. . Callan, T. McLoughlin and I. J. Swanson, "Holography beyond the Penrose limit," Nucl. Phys. B 694, 115 (2004), hep-th/0404007; C. G. . Callan, T. McLoughlin and I. J. Swanson, "Higher impurity AdS/CFT correspondence in the near-BMN limit," Nucl. Phys. B 700 (2004) 271, hep-th/0405153.

[17] D. Serban and M. Staudacher, "Planar N $=4$ gauge theory and the Inozemtsev long range spin chain," JHEP 0406, 001 (2004), hepth/0401057.

[18] N. Beisert, V. Dippel and M. Staudacher, "A novel long range spin chain and planar $\mathrm{N}=4$ super Yang-Mills," JHEP 0407 (2004) 075, hep-th/0405001.

[19] B. Eden, C. Jarczak and E. Sokatchev, "A three-loop test of the dilatation operator in N = 4 SYM," Nucl. Phys. B 712 (2005) 157, hepth/0409009.

[20] C. Sieg and A. Torrielli, Nucl. Phys. B 723 (2005) 3, hep-th/0505071.

[21] J. A. Minahan, "The SU(2) sector in AdS/CFT," Fortsch. Phys. 53 (2005) 828, hep-th/0503143. 
[22] N. Beisert, "The su(2|3) dynamic spin chain," Nucl. Phys. B 682 (2004) 487, hep-th/0310252.

[23] A. V. Belitsky, G. P. Korchemsky and D. Mueller, "Integrability in YangMills theory on the light cone beyond leading order," Phys. Rev. Lett. 94, 151603 (2005), hep-th/0412054; A. V. Belitsky, G. P. Korchemsky and D. Mueller, "Integrability of two-loop dilatation operator in gauge theories," hep-th/0509121.

[24] M. Staudacher, "The factorized S-matrix of CFT/AdS," JHEP 0505 (2005) 054, hep-th/0412188.

[25] N. Beisert and M. Staudacher, "Long-range PSU $(2,2 \mid 4)$ Bethe ansaetze for gauge theory and strings," hep-th/0504190.

[26] V. A. Kazakov and K. Zarembo, "Classical / quantum integrability in non-compact sector of AdS/CFT," JHEP 0410 (2004) 060, hepth/0410105; N. Beisert, V. A. Kazakov and K. Sakai, "Algebraic curve for the SO(6) sector of AdS/CFT," hep-th/0410253; S. Schafer-Nameki, "The algebraic curve of 1-loop planar $\mathrm{N}=4$ SYM," Nucl. Phys. B 714 (2005) 3, hep-th/0412254.

[27] G. Arutyunov, S. Frolov and M. Staudacher, "Bethe ansatz for quantum strings," JHEP 0410, 016 (2004), hep-th/0406256.

[28] S. Schafer-Nameki, M. Zamaklar and K. Zarembo, "Quantum corrections to spinning strings in $\operatorname{AdS}(5) \times \mathrm{S}^{* *} 5$ and Bethe ansatz: A comparative study," JHEP 0509 (2005) 051, hep-th/0507189.

[29] S. Schafer-Nameki and M. Zamaklar, "Stringy sums and corrections to the quantum string Bethe ansatz," hep-th/0509096.

[30] N. Beisert and A. A. Tseytlin, "On quantum corrections to spinning strings and Bethe equations," hep-th/0509084.

[31] I. R. Klebanov, M. Spradlin and A. Volovich, "New effects in gauge theory from pp-wave superstrings," Phys. Lett. B 548, 111 (2002), hepth/0206221.

[32] N. Mann and J. Polchinski, "Bethe ansatz for a quantum supercoset sigma model," Phys. Rev. D 72 (2005) 086002, hep-th/0508232. 
[33] V. V. Bazhanov, S. L. Lukyanov and A. B. Zamolodchikov, "Quantum field theories in finite volume: Excited state energies," Nucl. Phys. B 489 (1997) 487, hep-th/9607099.

[34] M. Lüscher, "On A Relation Between Finite Size Effects And Elastic Scattering Processes," DESY 83/116 Lecture given at Cargese Summer Inst., Cargese, France, Sep 1-15, 1983

[35] M. Lüscher, "Volume Dependence Of The Energy Spectrum In Massive Quantum Field Theories. 1. Stable Particle States," Commun. Math. Phys. 104 (1986) 177.

[36] T. D. Schultz, D. C. Mattis and E. H. Lieb, "Two-Dimensional Ising Model As A Soluble Problem Of Many Fermions," Rev. Mod. Phys. 36 (1964) 856.

[37] T. R. Klassen and E. Melzer, "Purely Elastic Scattering Theories And Their Ultraviolet Limits," Nucl. Phys. B 338 (1990) 485.

[38] T. R. Klassen and E. Melzer, "On The Relation Between Scattering Amplitudes And Finite Size Mass Corrections In Qft," Nucl. Phys. B 362 (1991) 329.

[39] G. Munster, "The Size Of Finite Size Effects In Lattice Gauge Theories," Nucl. Phys. B 249 (1985) 659.

[40] R. R. Metsaev, C. B. Thorn and A. A. Tseytlin, "Light-cone superstring in AdS space-time," Nucl. Phys. B 596 (2001) 151, hep-th/0009171.

[41] L. F. Alday, G. Arutyunov and S. Frolov, "New integrable system of 2 dim fermions from strings on $\operatorname{AdS}(5) \times \mathrm{S}^{* *} 5$," hep-th/0508140.

[42] A. B. Zamolodchikov, "Thermodynamic Bethe Ansatz In Relativistic Models. Scaling Three State Potts And Lee-Yang Models," Nucl. Phys. B 342, 695 (1990).

[43] C. Destri and H. J. De Vega, "Unified approach to thermodynamic Bethe Ansatz and finite size corrections for lattice models and field theories," Nucl. Phys. B 438, 413 (1995), hep-th/9407117. 
[44] See e.g. P. A. Pearce, L. Chim and C. Ahn, "Excited TBA Equations II: Massless Flow from Tricritical to Critical Ising Model," Nucl. Phys. B 660 (2003) 579, hep-th/0302093;

J. Balog and A. Hegedus, "TBA equations for the mass gap in the O(2r) non-linear sigma-models," Nucl. Phys. B 725, 531 (2005), hepth/0504186.

[45] P. Dorey and R. Tateo, "Excited states by analytic continuation of TBA equations," Nucl. Phys. B 482 (1996) 639, hep-th/9607167.

[46] P. Dorey and R. Tateo, "Excited states in some simple perturbed conformal field theories," Nucl. Phys. B 515 (1998) 575, hep-th/9706140.

[47] B.J.B. Crowley, "Some generalisations of the Poisson summation formula," J. Phys. A12 (1979) 1951.

[48] J. Balog and A. Hegedus, "TBA equations for excited states in the $\mathrm{O}(3)$ and $\mathrm{O}(4)$ nonlinear sigma-model," J. Phys. A 37 (2004) 1881, hep-th/0309009. 